\title{
Nonmigrating tidal signals in the upper thermospheric zonal wind at equatorial latitudes as observed by CHAMP
}

\author{
K. Häusler and H. Lühr \\ GFZ German Research Centre for Geosciences, Telegrafenberg, 14473 Potsdam, Germany \\ Received: 1 October 2008 - Revised: 24 June 2009 - Accepted: 24 June 2009 - Published: 3 July 2009
}

\begin{abstract}
The accelerometer onboard CHAMP enables us to derive the thermospheric zonal wind at orbit altitudes $(\sim 400 \mathrm{~km})$. Numerous equatorial overflights $(\sim 45250)$ are used to investigate the influence of nonmigrating tides on the thermospheric zonal wind. In a previous study a so called "wave-4" longitudinally signal observed in the satellite frame was identified in the zonal wind residuals during equinox. Using four years of data (2002-2005), we determine the annual variation of this prominent feature which is strongest during the months of July through October and has a smaller second maximum during March/April. Due to the large data set we were able to separate the observed wavenumbers into the tidal components. Thereby, we can identify the eastward propagating diurnal tide with zonal wavenumber $s=3$ (DE3) as the prime cause for the observed wave-4 pattern in the zonal wind. Analyzing the zonal wind along the geographic and the dip equator revealed that the largest amplitudes of DE3 are found along the dip equator. Besides DE3 we present the full spectrum of nonmigrating tides in the upper thermosphere.
\end{abstract}

Keywords. Meteorology and atmospheric dynamics (Thermospheric dynamics; Waves and tides)

\section{Introduction}

Knowing the characteristics of the zonal wind in the upper thermosphere is a key issue for the understanding of the electrodynamics of the upper atmosphere due to ion/neutral interaction. Since its launch in July 2000, the global and continuous measurements of the satellite CHAMP (CHAllenging Minisatellite Payload) contribute considerably to the understanding of the zonal wind. In a first study, Liu et al. (2006)

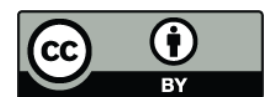

Correspondence to: K. Häusler (kathrin@gfz-potsdam.de) presented a climatology of CHAMP zonal winds in the equatorial region. The authors confirmed the expected dominant diurnal variation with westward winds in the morning sector and eastward winds in the evening sector. Furthermore, the zonal wind revealed a strong dependence on solar flux, but little dependence on magnetic activity. In a statistical analysis, Häusler et al. (2007) investigated the longitudinal dependence of the CHAMP zonal delta winds (deviations from the zonal average) at dip equator latitudes for the combined equinoxes as well as for June and December solstices. In that study, the largest longitudinal dependences are observed during the morning hours, 03:00-09:00 local time (LT). In this local time sector the direction of the delta wind is reversing sign between the two solstices, and the equinoxes are exhibiting the switch in polarity between the two solstices. The influence of the solar flux level was found to be insignificant. However, performing a Fourier transform of the equinox data revealed the dominance of a wavenumber 4 longitudinally structure in some LT sectors. The wave-4 pattern is a prevailing feature specifically observed in the slowly precessing satellite frame.

Numerous studies based on satellite data have reported a wave-4 structure in several atmospheric parameters. This fascinating feature of the atmosphere was identified in the electron density (e.g., Lin et al., 2007; Lühr et al., 2007), electric field (e.g., Hartman and Heelis, 2007; Kil et al., 2007), electrojet (e.g., England et al., 2006; Lühr et al., 2008) as well as in ionospheric emissions (Sagawa et al., 2005; Immel et al., 2006) but also in the neutral parameters of the atmosphere like MLT (Mesosphere Lower Thermosphere) temperatures (Forbes et al., 2006). In the MLT region the basic wave responsible for the wave- 4 pattern is said to be the eastward propagating diurnal tide with zonal wavenumber 3 (DE3). This wave is primarily excited by latent heat release in the tropical troposphere (Hagan and Forbes, 2002).

In this work, we address the open issue of how the detected wave- 4 structure in the upper thermospheric zonal

Published by Copernicus Publications on behalf of the European Geosciences Union. 
Table 1. The listed diurnal (D) and semidiurnal (S) oscillations propagating either eastward (E) or westward (W) with a given zonal wavenumber, $s$, or remain standing $(0)$ as well as stationary planetary waves ( $\mathrm{sPW} m$ ) can account for the observed wave- $m$ structures in satellite data taken at a quasi constant LT.

\begin{tabular}{cll}
\hline observed & & contributing oscillations \\
\hline wave-1 & $\longrightarrow$ & DW2, D0, SW3, SW1, sPW1 \\
wave-2 & $\longrightarrow$ & DW3, DE1, SW4, S0, sPW2 \\
wave-3 & $\longrightarrow$ & DW4, DE2, SW5, SE1, sPW3 \\
wave-4 & $\longrightarrow$ & DW5, DE3, SW6, SE2, sPW4 \\
\hline
\end{tabular}

wind behaves over the course of a year and whether it can be attributed to the DE3 tidal signal in the MLT region. Furthermore, we present the diurnal and semidiurnal tidal spectra of the zonal wind within a latitude band both along the geographic and the dip equator which indicate the presence of other nonmigrating tides. For our analysis we make use of 4 years of CHAMP accelerometer measurements taken between January 2002 through December 2005.

\section{Atmospheric tides}

Global-scale oscillations in temperature, wind, and density with periods that are harmonics of a solar day are referred to as atmospheric tides whereas one distinguishes between "migrating" and "nonmigrating" tides (e.g., Chapman and Lindzen, 1970; Forbes, 1995). Migrating tides propagate westward with the apparent motion of the sun and are thus sun-synchronous and longitude independent when observed at constant local time. Thereby, the zonal wavenumber, $s$, is equal to the frequency (in cycles per day). Migrating tides are prompted primarily by the absorption of solar energy by tropospheric water and water vapor, as well as stratospheric ozone (Oberheide et al., 2002). Nonmigrating tides are excited for instance by zonal asymmetries (i.e. topography, land-sea differences, longitude dependences in absorbing species) (Forbes et al., 2003) or by nonlinear interactions between the migrating diurnal tide and planetary waves (Hagan and Roble, 2001) or gravity waves (McLandress and Ward, 1994). Another important source of nonmigrating tides and denotative for the observed wave-4 structure is latent heat release in the tropical troposphere (Hagan and Forbes, 2002). The zonal wavenumber, $s$, of nonmigrating tides is not equal to the frequency (in cycles per day) which means that they can either propagate eastward (E) $(s<0)$, westward (W) $(s>0)$ or remain standing $(s=0)$.

Atmospheric tides can be expressed, in general, in the form

$A_{n, s} \cos \left(n \Omega t+s \lambda-\phi_{n, s}\right)$

where $A_{n, s}=$ amplitude, $n$ denotes a subharmonic of a solar day, $\Omega=$ rotation rate of the Earth, $t=$ universal time, $s$ is the above mentioned zonal wavenumber, $\lambda=$ longitude, and $\phi_{n, s}$ $=$ phase (Forbes et al., 2006).

Oscillations with periods of $24 \mathrm{~h}$ and $12 \mathrm{~h}$ are referred to as diurnal (D) and semidiurnal (S) tides and correspond to $n=1$, 2 , respectively. Throughout this paper we will use the notation DWs and DEs to describe a westward or eastward propagating diurnal tide with zonal wavenumber $s$. For semidiurnal tides $D$ will be replaced by $S$ and the standing oscillations are termed D0 and S0. Stationary planetary waves with zonal wavenumber $m$ are denoted as sPWm. The phase is defined as the universal time (UT) when the maximum passes the zero degree longitude, i.e. the local time at the Greenwich meridian.

CHAMP is orbiting the Earth on a circular, near polar orbit at an inclination of $87.3^{\circ}$. The satellite precesses through one hour of local time every 11 days, thus taking its daily measurements at a quasi constant local time. Converting Eq. (1) from universal time to a local time frame by using $t=t_{L T}-\lambda / \Omega$, we get

$A_{n, s} \cos \left(n \Omega t_{L T}+(s-n) \lambda-\phi_{n, s}\right)$

Looking at Eq. (2), we see firstly why migrating tides $(s=n)$ are longitude independent $(s-n=0)$ in the local time frame, secondly that an observed wave- 4 structure in satellite data cannot be related unambiguously to a certain tidal component because it is consistent with any values of $s$ and $n$ for which holds $|s-n|=4$. In other words, the observed wave-4 pattern can be caused by a diurnal tide $(n=1)$ with $s=-3$ or $s=+5$, a semidiurnal tide $(n=2)$ with $s=-2$ or $s=+6$, or by a stationary planetary wave $(n=0)$ with $s=4$. Table 1 summarizes the individual contributions to the observed wave structures in satellite data. The individual contributions to the observed wave- 4 can, however, be discriminated by their local time evolution. Within $24 \mathrm{~h} \mathrm{LT}$, a wave- 4 structure caused by DW5 propagates $90^{\circ}$ to the west while DE3 propagates $90^{\circ}$ to the east; SW6 displays a phase shift of $180^{\circ}$ to the west while SE2 exhibits $180^{\circ}$ to the east; sPW4 shows no phase shift at all.

In order to quantify the responsible oscillations for the wave- 4 structure, we have to identify the individual contributions. This can be done not only by looking at the phase propagation of the observed wave- 4 but also by performing a two-dimensional (2-D) Fourier transform with the data.

\section{Data set and method of analysis}

The data set employed in this study is comparable to the one used by Häusler et al. (2007). However, in this work we have extended the time period used in Häusler et al. (2007) to include all years from 2002-2005. Four years of CHAMP data are needed to cover each month of the year with $24 \mathrm{~h}$ of LT. The $24 \mathrm{~h}$ LT coverage is a mandatory requirement in order to perform the 2-D Fourier transform. As already described in the previous section, CHAMP precesses through 
one hour of local time every 11 days. Combining the ascending and descending node of the satellite, $24 \mathrm{~h}$ local time coverage is obtained after approximately 131 days. For our statistical analysis, there are roughly 45250 CHAMP overflights available. The distribution of these equator passes is depicted in Fig. 1 where one can identify the precession of the CHAMP orbit through local time as well. It is also visible that 4 years of data do not provide a homogeneous $24 \mathrm{~h}$ LT coverage. For each month/LT hour bin, there are between 200 and 700 passes available. However, for each LT hour overlapping $3 \mathrm{~h}$ bins of local time centered around the hour of interest are used in order to get a sufficient amount of data for each bin for further processing.

Within this study we are investigating the zonal delta wind derived from CHAMP accelerometer measurements as previously introduced by Häusler et al. (2007). The zonal delta winds are wind residuals from which the zonal mean was removed. For further description on how to derive the zonal wind from CHAMP accelerometer readings see Liu et al. (2006), and for the zonal wind residual calculation see Häusler et al. (2007).

Furthermore, we concentrate only on data taken between $10^{\circ} \mathrm{N}$ and $10^{\circ} \mathrm{S}$ along the geomagnetic equator because that is where the wave-4 structure in the zonal wind was first identified. Later on, for comparison, we will also survey data taken between $10^{\circ} \mathrm{N}$ and $10^{\circ} \mathrm{S}$ along the geographic equator.

After sorting the wind readings into 24 overlapping longitude bins of $30^{\circ}$ width, we can perform the first Fourier transform which will bring forth the observed wavenumbers in satellite data. The observed longitudinal variation of the zonal wind velocity for a given wavenumber, $m$, can be calculated for each month and LT by inserting the determined Fourier coefficients $a$ and $b$ into the following equation

wave- $m(\lambda)=a_{m} \cos (m \lambda)+b_{m} \sin (m \lambda)$

with $m=1,2,3,4$.

As already discussed in Sect. 2, the wavenumber, $m$, observed by a satellite moving slowly in local time can be caused by several tidal components. This ambiguity can be expressed mathematically (Lühr et al., 2008) as

$$
\begin{aligned}
A_{m} \cos (m \lambda+\varphi)= & a_{n-m} \cos \left\{m \lambda-\frac{\pi n}{12}\left(\mathrm{LT}-t_{n-m}\right)\right\} \\
& +a_{m} \cos \left\{m \lambda-\frac{\pi n}{12} t_{m}\right\} \\
& +a_{n+m} \cos \left\{m \lambda+\frac{\pi n}{12}\left(\mathrm{LT}-t_{n+m}\right)\right\},
\end{aligned}
$$

where $A_{m}$ is the amplitude of the total $m$-th harmonic, $\lambda$ is the longitude, $\varphi$ a local time dependent phase shift, $n(=1,2)$ denotes harmonics of the once per day oscillation, $a_{n-m}, a_{m}$, $a_{n+m}$ are the amplitudes of the various tidal components and stationary planetary waves, and $t_{n-m}, t_{m}, t_{n+m}$ are the corresponding phases, i.e. the universal time at which the maximum is passing the Greenwich meridian; for the stationary

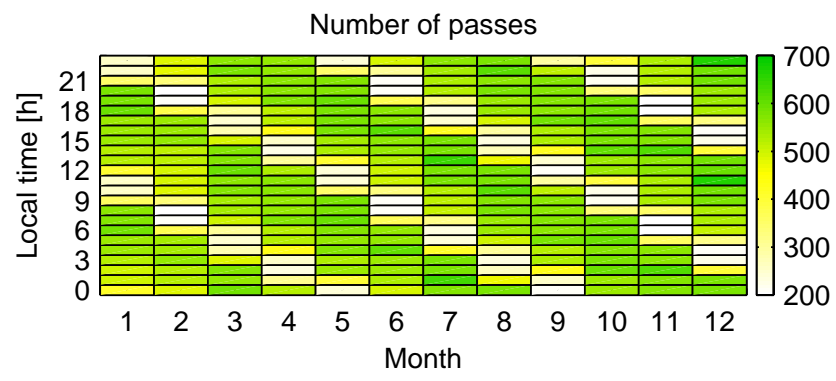

Fig. 1. Number of passes used in the statistical analysis for each month/local time hour bin.

planetary waves the longitude of the maximum. In cases where the signal is available from all longitudes and local times, Eq. (4) can be solved analytically for $a_{n-m}, a_{m}, a_{n+m}$ and $t_{n-m}, t_{m}, t_{n+m}$ by performing a second Fourier transform for every combination of $n$ and $m$ and thus revealing all the tidal and stationary planetary wave contributions.

\section{Results}

One of the open issues we want to discuss within this paper is the annual variation of the observed wave- 4 structure in the zonal wind at $400 \mathrm{~km}$ altitude. Häusler et al. (2007) found a dominating wave-4 structure at equinoxes during some local times along the dip equator. Performing a Fourier transform of the longitudinally binned data, as described in Sect. 3, yields the observed wavenumbers summarized in Table 1 .

When taking the results of the Fourier transform for the fourth harmonic and calculating with the help of Eq. (3) the longitudinal signal distribution versus local time, we get the annual variation of the wave-4 as displayed in Fig. 2. Depicted in Fig. 2 is the wave- 4 structure for each month of the year. We see that the wave-4 is present throughout the year with varying strength and phase properties. The strongest wave-4 signals are found in the months of July through September. During these months, the wave-4 pattern is propagating $90^{\circ}$ to the east within $24 \mathrm{~h} \mathrm{LT}$ as indicated by the black guiding line. As already discussed in Sect. 2 and illustrated by Lühr et al. (2008) in their Fig. 1, a phase propagation of $90^{\circ}$ to the east within $24 \mathrm{~h} \mathrm{LT}$ would be induced by the nonmigrating tide DE3. Thus, suggesting DE3 to be the cause for the observed wave-4 structure in the zonal wind at CHAMP altitude. The months of March and April as well as October and to some extent also November display the $90^{\circ}$ phase propagation to the east although with reduced amplitudes. But for the months of December through February, there is no clear phase propagation recognizable and also the amplitudes are rather small compared to the dominating boreal summer months. However, when looking at Fig. 2, it is also noticeable that the strength of the wave- 4 is varying with LT. For example, in July the biggest amplitude of $27.5 \mathrm{~m} / \mathrm{s}$ is 

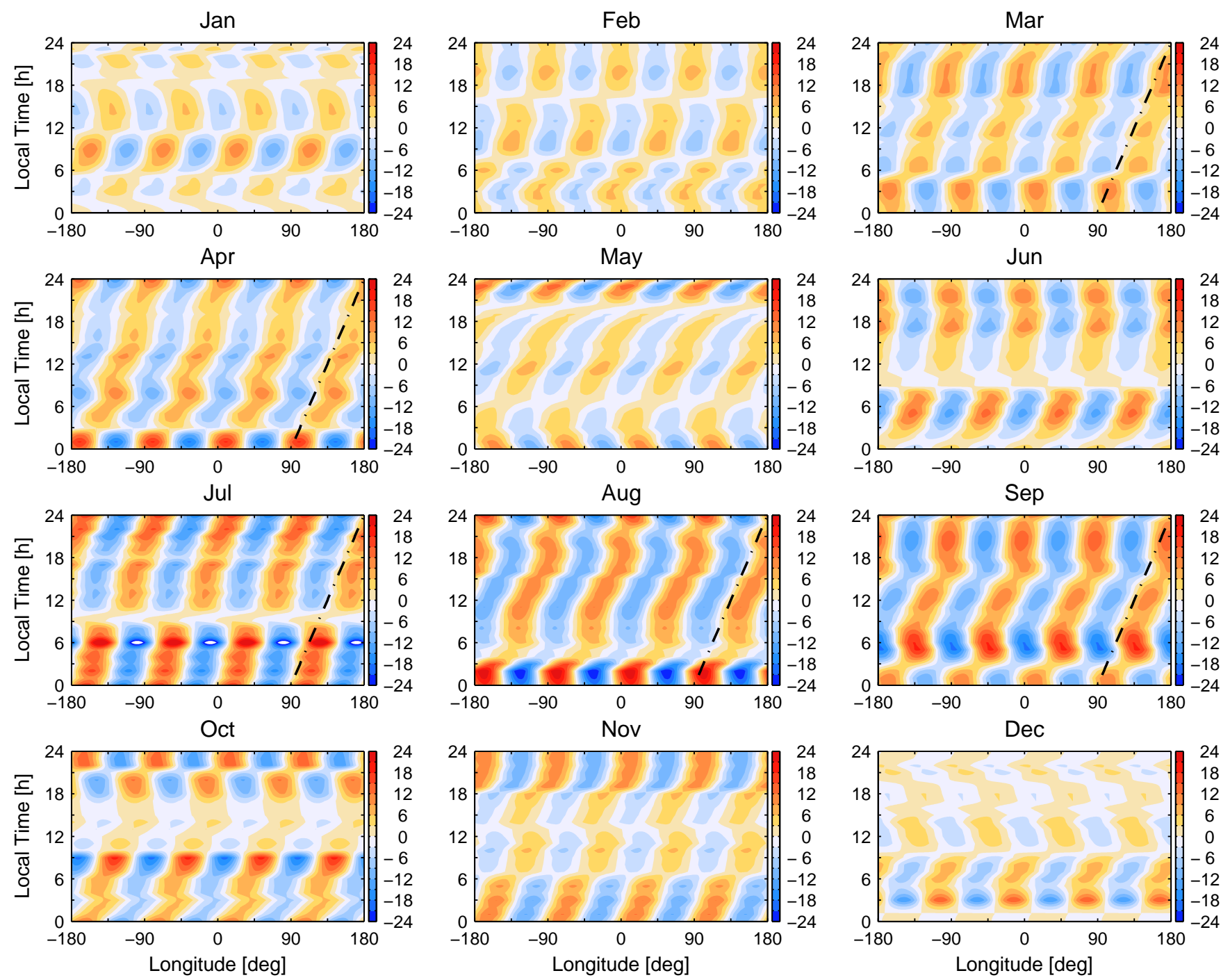

Fig. 2. Longitude vs. local time distribution of the observed wave- 4 structure in the zonal wind [m/s] for each month of the year. The black guiding line is indicating a phase propagation of $90^{\circ}$ to the east within $24 \mathrm{~h} \mathrm{LT}$.

found at 06:00 LT, while already at 10:00 LT the amplitude decreases to $1.2 \mathrm{~m} / \mathrm{s}$. Thereafter it increases again to $15.5 \mathrm{~m} / \mathrm{s}$ at 22:00 LT. The observed LT variations can be attributed to the cumulative effect of the different tidal components contributing to the wave-4.

After identifying the annual variation of the wave- 4 structure, the remaining question is which oscillations are causing this pattern. In order to answer this question it is necessary according to Sect. 3 to perform a second Fourier transform. Figure 3 shows the results of the second Fourier transform for the observed wave- 4 and thus displays over the course of a year amplitude variations of the waves that are responsible for it; namely DW5, DE3, SW6, SE2, and sPW4 pursuant to Table 1. Looking at Fig. 3 it is obvious that DE3 is dominating by far the other components throughout the year and hence confirming the assumption that this nonmigrating tide is predominantly responsible for the observed wave- 4 structure in the zonal wind at $400 \mathrm{~km}$ altitude. All the other contributing waves are rather weak oscillations hardly exceeding $3 \mathrm{~m} / \mathrm{s}$ while DE3 is exhibiting two maxima, one from July through September and a smaller one in March/April. We find mean amplitudes for March and April of $6.7 \mathrm{~m} / \mathrm{s}$ and $7.2 \mathrm{~m} / \mathrm{s}$, respectively which are comparable to the ones in October/November. The biggest amplitude of DE3 is reached in July with $10.5 \mathrm{~m} / \mathrm{s}$ followed by $9.6 \mathrm{~m} / \mathrm{s}$ in August and September. From December through February, DE3 is rather weak with amplitudes that are not exceeding $4 \mathrm{~m} / \mathrm{s}$. During that time of the year, the strength of DE3 is comparable to the other contributions to the wave- 4 structure and hence explains why during that time no clear phase propagation is recognizable. 
However, besides the much discussed wave-4 structure, we also observe wavenumbers with $m=1,2,3$. Applying the above described analysis approach and calculating the corresponding oscillations for $m=1,2,3,4$ and $n=1,2$, we get the diurnal and semidiurnal tidal spectrum embedded in the zonal wind at CHAMP altitude. The tidal spectra were calculated both along the geomagnetic and the geographic equator within the latitude range of $\pm 10^{\circ}$. Figure 4 shows the obtained tidal spectra for both coordinate systems as well as the stationary planetary waves over the course of the year. Due to the data taking by the satellite and the processing approach, the migrating tides are filtered out and hence do not appear in Fig. 4.

When looking at the diurnal tidal spectra in Fig. 4, it is noticeable that overall larger amplitudes are observed along the geomagnetic equator (top left panel). We find the strongest amplitudes of the tidal spectrum in December/January for DW2 with an amplitude of $12.0 \mathrm{~m} / \mathrm{s}$ and $14.3 \mathrm{~m} / \mathrm{s}$, respectively. Along the geographic equator (top right panel), the amplitudes of DW2 for December/January are reduced to $5.3 \mathrm{~m} / \mathrm{s}$ and $7.7 \mathrm{~m} / \mathrm{s}$, respectively. Another strong tidal component observed along the geomagnetic equator is the standing diurnal oscillation D0 peaking in December with an amplitude of $11.3 \mathrm{~m} / \mathrm{s}$ which almost vanishes when considered in geographic coordinates.

An interesting result is revealed for the annual behavior of the DE2 and DE3 tides within both coordinate systems. Therefore, the annual variations of DE2 and DE3 are replotted in Fig. 5. It is striking that except for the months December and January the amplitudes of DE3 are clearly bigger along the geomagnetic equator than along the geographic equator. The annual variation is the same, just the amplitudes are reduced by $1.0 \mathrm{~m} / \mathrm{s}$ in November and $3.1 \mathrm{~m} / \mathrm{s}$ in May when analyzed in geographic coordinates. While DE2 has roughly the same amplitudes from September through December and for February and March within the both coordinate systems, the amplitudes from April to August are larger within the geographic frame than in the geomagnetic frame (between $1.8 \mathrm{~m} / \mathrm{s}$ in August and $2.4 \mathrm{~m} / \mathrm{s}$ in May). In January, the amplitude of DE2 is $1.6 \mathrm{~m} / \mathrm{s}$ higher in geomagnetic coordinates.

The semidiurnal tidal spectra in Fig. 4 are dominated by SW3, SW1, and SE1. While SW3 is displaying generally larger amplitudes along the geographic equator (middle right panel) peaking with an amplitude of $9.5 \mathrm{~m} / \mathrm{s}$ in August, SW1 is showing essentially larger amplitudes along the geomagnetic equator (middle left panel) peaking with an amplitude of $7.7 \mathrm{~m} / \mathrm{s}$ in December. Incidentally for December, the biggest amplitude of SW1 with $9.0 \mathrm{~m} / \mathrm{s}$ is observed in geographic coordinates exceeding the geomagnetic amplitude by $1.3 \mathrm{~m} / \mathrm{s}$. However, independent of the coordinate system, SW3 and SW1 have maxima in the same months. The same is true for SE1 which is usually stronger along the geomagnetic equator. However, it is noticeable that SE1 is peaking during the same months as DE2.

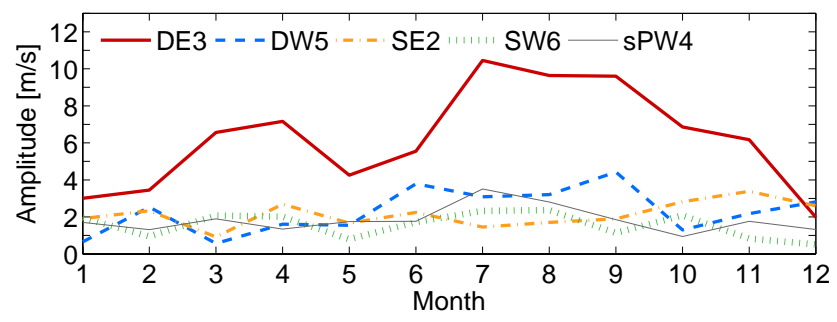

Fig. 3. Amplitude of the diurnal (DE3, DW5), semidiurnal (SE2, SW6) and stationary (sPW4) components contributing to the observed wave-4 structure in the zonal wind along the dip equator versus month of the year. DE3 is the most prominent component throughout the year and thus responsible for the observed wave- 4 structure.

The stationary planetary waves are displayed in the bottom panels of Fig. 4. Looking at sPW1, it is observable that this oscillation is peaking during solstices. While for June solstice the amplitudes between the two coordinate systems are of comparable strength, the amplitudes for December solstice are almost twice as high along the geomagnetic equator (bottom left panel) compared to the geographic equator (bottom right panel). Like SE1, sPW3 is disclosing maxima at the time of strong DE2 peaking in November with an amplitude of $6.0 \mathrm{~m} / \mathrm{s}$ and $5.8 \mathrm{~m} / \mathrm{s}$ along the geomagnetic and geographic equator, respectively. It is further noteworthy that $\mathrm{SPW} 3$ is showing the same variations between the two coordinate systems as DE2, namely being stronger from March through August in the geographic reference frame and stronger for the remaining year in the geomagnetic reference frame. The amplitudes and phases for the just discussed oscillations are listed in Table 2 for a latitude strip along the geomagnetic as well as the geographic equator.

\section{Discussion}

Within this section we want to discuss our findings in the context of previous results related to nonmigrating tides in the upper atmosphere. But first we turn our attention to the reliability of our results. Liu et al. (2006) name an uncertainty of $20 \mathrm{~m} / \mathrm{s}$ for the zonal wind calculated from CHAMP accelerometer measurements. However, we don't consider the total zonal wind velocity but zonal wind residuals, i.e. the zonal mean was removed. Investigating the longitudinal dependence of the zonal wind, Häusler et al. (2007) found that the biggest standard deviations for the longitudinal binned zonal wind residuals are found in the early morning hours when the thermosphere has its lowest density of the day. In this study we use the same processing steps to get to the longitudinally binned data. Thus the same variability of the zonal wind residuals can be assumed. In order to test the reliability of our method calculating the individual tidal components (a similar approach is used by Lowes and Olsen, 2004) 

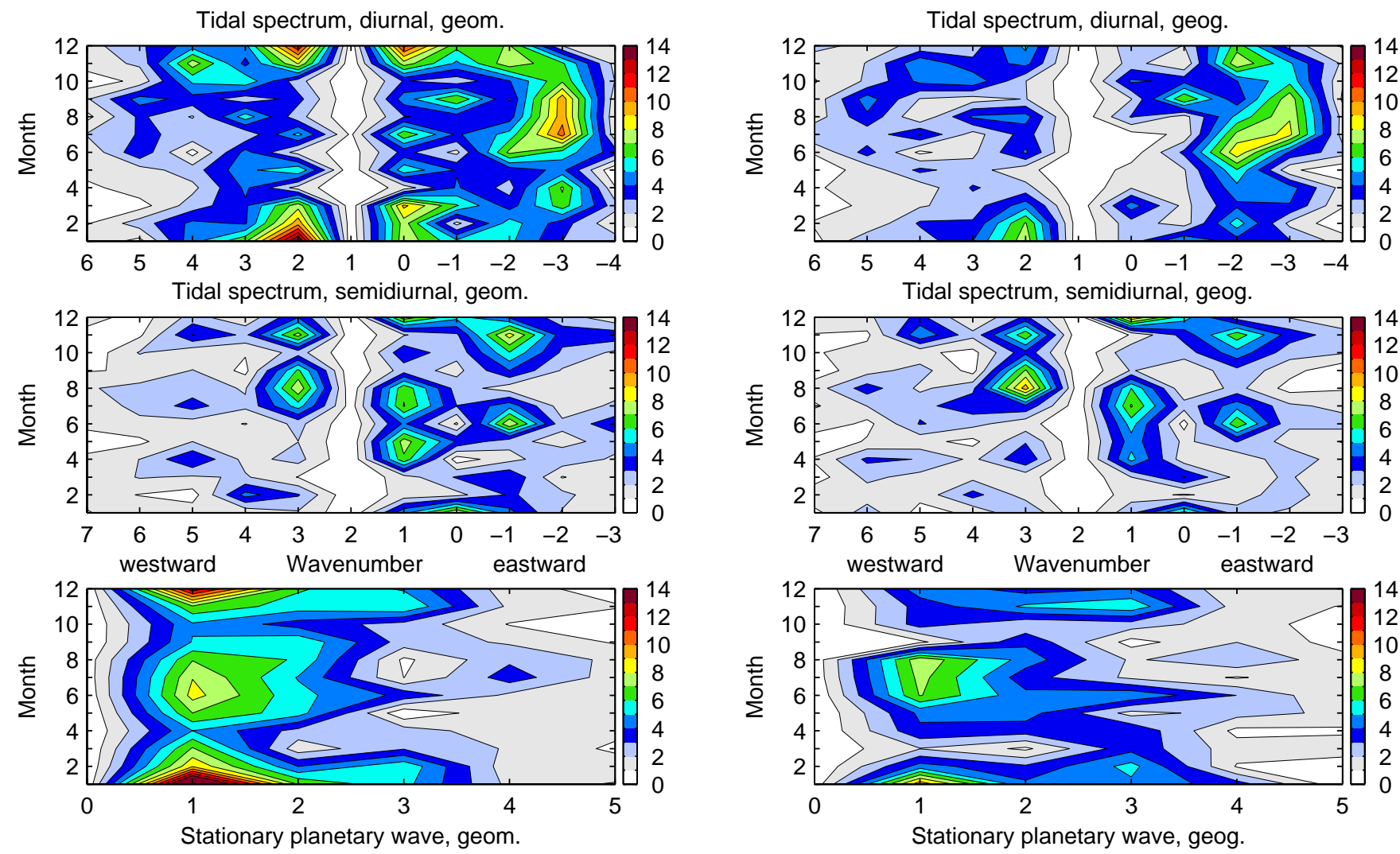

Fig. 4. Diurnal (top) and semidiurnal (middle) tidal spectra of the zonal wind $[\mathrm{m} / \mathrm{s}]$ along the geomagnetic equator (left panels) and the geographic equator (right panels). Bottom panels display the stationary planetary waves.

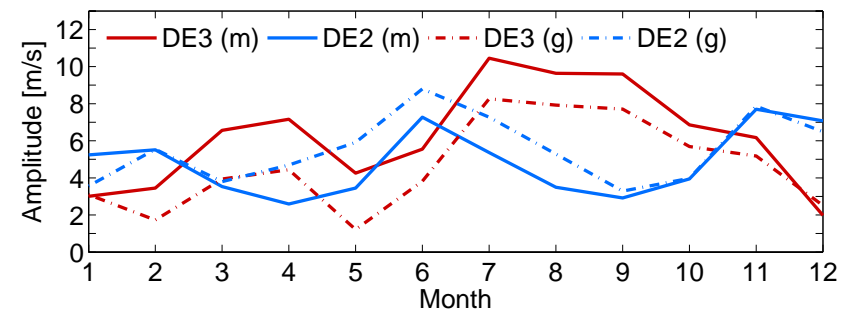

Fig. 5. Comparison of DE3 (red) and DE2 (blue) tides at the geomagnetic (solid) and at the geographic (dash-dotted) equator. The biggest amplitudes of DE3 are reached along the geomagnetic equator while DE2 is peaking along the geographic equator.

we rerun our processing approach 100 times using subsets of $25 \%, 35 \%, \ldots, 85 \%, 95 \%$ of our available data. Thereby, the 100 individual data sets for each processing run were selected randomly out of the existing data. After this we calculated the standard deviation of the 100 individual results for each tidal component and subset. The standard deviations are decreasing continuously from the $25 \%$ to the $95 \%$ data subsets. Extrapolating to $100 \%$ yields the estimated uncertainties of

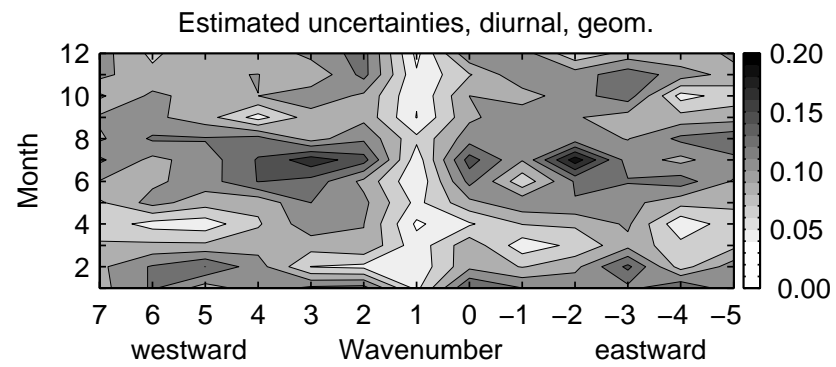

Fig. 6. Estimated formal uncertainties $[\mathrm{m} / \mathrm{s}]$ of the CHAMP tidal analysis for the diurnal tidal spectrum along the geomagnetic equator. The largest uncertainties are observed during June Solstice when the upper thermospheric density has its minimum.

our method. Figure 6 displays the estimated uncertainties for the diurnal tidal wind spectrum along the geomagnetic equator as resulting from the statistical test. We see that the estimated formal uncertainties are between $0.1 \mathrm{~m} / \mathrm{s}$ and $0.2 \mathrm{~m} / \mathrm{s}$ with largest values during June solstice. This again can be related to the reduced thermospheric density. During the middle of the year the air density attains its minimum 
Table 2. Zonal wind tidal amplitudes [m/s] (top) and phases [UT] (bottom) observed with CHAMP at $400 \mathrm{~km}$ altitude along the geomagnetic/geographic equator. The last two columns contain the amplitudes [m/s] (top) and longitudes of maximum (bottom) for the stationary planetary waves sPW1 and sPW3. The damping of the amplitude due to the binning procedure as discussed in Sect. 5 is considered.

\begin{tabular}{|c|c|c|c|c|c|c|c|c|c|}
\hline month & DW2 & D0 & DE2 & DE3 & SW3 & SW1 & SE1 & sPW1 & sPW3 \\
\hline 1 & $14.3 / 7.7$ & $7.7 / 2.6$ & $5.2 / 3.6$ & $3.0 / 3.1$ & $1.9 / 3.0$ & $4.7 / 2.8$ & $4.3 / 2.4$ & $16.7 / 9.8$ & $5.3 / 4.7$ \\
\hline 2 & $9.6 / 7.1$ & $7.5 / 2.6$ & $5.5 / 5.5$ & $3.5 / 1.7$ & $3.1 / 1.5$ & $1.4 / 1.5$ & $3.1 / 1.5$ & $8.9 / 4.4$ & $5.8 / 5.4$ \\
\hline 3 & 7.6/4.6 & $9.3 / 4.5$ & $3.5 / 3.8$ & $6.6 / 3.9$ & $1.0 / 1.4$ & $1.5 / 2.0$ & $3.9 / 1.7$ & $7.1 / 2.0$ & $2.9 / 3.7$ \\
\hline 4 & $1.6 / 2.3$ & $1.4 / 2.0$ & $2.6 / 4.7$ & $7.2 / 4.4$ & $2.3 / 3.8$ & $6.7 / 5.4$ & $1.7 / 1.3$ & $5.0 / 3.8$ & $2.5 / 3.9$ \\
\hline 5 & $5.9 / 1.3$ & $5.7 / 1.4$ & $3.5 / 5.9$ & $4.3 / 1.2$ & $2.0 / 2.9$ & $7.5 / 4.6$ & $2.3 / 3.0$ & $7.0 / 4.2$ & $0.2 / 1.7$ \\
\hline 6 & $1.6 / 4.2$ & $3.3 / 0.4$ & 7.3/8.8 & $5.6 / 3.8$ & $2.3 / 1.3$ & $4.2 / 5.4$ & $7.9 / 6.8$ & $8.4 / 7.1$ & $3.7 / 4.8$ \\
\hline 7 & $5.3 / 3.4$ & $6.8 / 0.6$ & $5.4 / 7.3$ & $10.5 / 8.3$ & $5.5 / 3.8$ & $7.1 / 7.1$ & $4.6 / 4.6$ & $8.0 / 7.4$ & $1.0 / 1.8$ \\
\hline 8 & $2.3 / 4.4$ & $2.9 / 3.1$ & $3.5 / 5.3$ & $9.6 / 7.9$ & $7.8 / 9.5$ & $6.7 / 6.0$ & $1.5 / 2.3$ & $6.9 / 7.7$ & $0.8 / 1.9$ \\
\hline 9 & $3.3 / 2.1$ & $4.5 / 2.0$ & $2.9 / 3.3$ & $9.6 / 7.7$ & $6.2 / 7.1$ & $2.5 / 2.1$ & $4.0 / 2.7$ & $5.1 / 0.8$ & $2.6 / 0.6$ \\
\hline 10 & $2.4 / 2.0$ & $3.1 / 4.2$ & $4.0 / 4.0$ & $6.9 / 5.7$ & $2.4 / 3.7$ & $3.7 / 2.9$ & $5.9 / 4.6$ & $4.9 / 3.4$ & $2.6 / 2.5$ \\
\hline 11 & $8.1 / 4.4$ & $7.6 / 2.4$ & 7.7/7.9 & $6.2 / 5.2$ & $7.4 / 6.8$ & $1.9 / 1.1$ & $8.5 / 6.6$ & $6.8 / 3.4$ & $6.0 / 5.8$ \\
\hline 12 & $12.0 / 5.3$ & $11.3 / 2.7$ & $7.1 / 6.5$ & $2.0 / 2.5$ & $3.9 / 3.2$ & $7.7 / 9.0$ & $3.8 / 2.4$ & $12.8 / 4.6$ & $4.9 / 4.0$ \\
\hline month & DW2 & D0 & DE2 & DE3 & SW3 & SW1 & SE1 & sPW1 & sPW3 \\
\hline 1 & $11 / 11$ & $1 / 10$ & $6 / 7$ & $3 / 4$ & $11 / 2$ & $3 / 2$ & $7 / 7$ & $50^{\circ} / 40^{\circ}$ & $300^{\circ} / 280^{\circ}$ \\
\hline 2 & $11 / 11$ & $1 / 3$ & $5 / 6$ & $0 / 0$ & $10 / 0$ & $7 / 10$ & $6 / 6$ & $40^{\circ} / 30^{\circ}$ & $270^{\circ} / 270^{\circ}$ \\
\hline 3 & $12 / 12$ & $1 / 3$ & $7 / 6$ & $20 / 20$ & $10 / 1$ & $4 / 3$ & $6 / 6$ & $40^{\circ} / 30^{\circ}$ & $270^{\circ} / 240^{\circ}$ \\
\hline 4 & $18 / 12$ & $6 / 5$ & $4 / 6$ & $20 / 19$ & $3 / 3$ & $8 / 8$ & $5 / 4$ & $80^{\circ} / 110^{\circ}$ & $260^{\circ} / 240^{\circ}$ \\
\hline 5 & $23 / 4$ & $10 / 5$ & $5 / 6$ & $20 / 17$ & $12 / 10$ & $10 / 10$ & $5 / 6$ & $250^{\circ} / 240^{\circ}$ & $300^{\circ} / 280^{\circ}$ \\
\hline 6 & $1 / 8$ & $11 / 8$ & $6 / 6$ & $18 / 17$ & $5 / 3$ & $7 / 6$ & $5 / 5$ & $210^{\circ} / 180^{\circ}$ & $340^{\circ} / 320^{\circ}$ \\
\hline 7 & $0 / 10$ & $13 / 18$ & $3 / 5$ & $20 / 19$ & $6 / 6$ & $8 / 8$ & $5 / 4$ & $210^{\circ} / 190^{\circ}$ & $210^{\circ} / 270^{\circ}$ \\
\hline 8 & $19 / 13$ & $14 / 21$ & $3 / 5$ & $20 / 19$ & $11 / 11$ & $8 / 7$ & $3 / 3$ & $200^{\circ} / 180^{\circ}$ & $150^{\circ} / 240^{\circ}$ \\
\hline 9 & $9 / 10$ & $21 / 20$ & $5 / 7$ & $20 / 19$ & $9 / 8$ & $8 / 11$ & $6 / 6$ & $50^{\circ} / 320^{\circ}$ & $350^{\circ} / 260^{\circ}$ \\
\hline 10 & $6 / 1$ & $20 / 13$ & $7 / 8$ & $19 / 19$ & $5 / 4$ & $2 / 2$ & $6 / 6$ & $20^{\circ} / 290^{\circ}$ & $320^{\circ} / 300^{\circ}$ \\
\hline 11 & $12 / 14$ & $1 / 6$ & $6 / 5$ & $20 / 19$ & $10 / 10$ & $11 / 11$ & $5 / 5$ & $20^{\circ} / 340^{\circ}$ & $300^{\circ} / 290^{\circ}$ \\
\hline 12 & $13 / 16$ & $1 / 3$ & $6 / 5$ & $6 / 5$ & $9 / 8$ & $4 / 3$ & $6 / 6$ & $60^{\circ} / 40^{\circ}$ & $320^{\circ} / 30^{\circ}$ \\
\hline
\end{tabular}

(e.g. Müller et al., 2009), which causes a larger scatter of the wind data. The uncertainties of the other tidal spectra display variations comparable to the one shown in Fig. 6.

Another fact to be taken into account is the damping due to the data processing. The damping can be evoked on the one hand by the large longitudinal bins of $30^{\circ}$ width, and on the other hand by the binning of the local time into $3 \mathrm{~h} \mathrm{LT} \mathrm{com-}$ posites. Running our analyzing routines with test data sets revealed that the damping is following the well-know function $x^{-1} \sin x$. Considering the damping due to the longitude bins, we find that $x=\pi / 12, \pi / 6, \pi / 4, \pi / 3$ for the wave-1, wave- 2 , wave- 3 , wave- 4 , respectively. This leads to an underestimation of the corresponding tides due to the longitude bins by approximately $1 \%, 4 \%, 10 \%, 17 \%$ observed as wave1 , wave- 2 , wave- 3 , and wave- 4 , respectively. Regarding the local time binning, we find $x=\pi / 8$ and $\pi / 4$ for the diurnal and semidiurnal tides yielding an undervaluation of the diurnal and semidiurnal tides by $3 \%$ and $10 \%$, respectively. The total damping is the product between the two contributions. Therefore, the diurnal tides observed as wave-1, wave2 , wave- 3 , and wave- 4 are underestimated by our approach by $4 \%, 7 \%, 13 \%$, and $20 \%$, respectively. The semidiurnal tides observed as wave- 1 , wave- 2 , wave- 3 , and wave- 4 are underestimated by $11 \%, 14 \%, 19 \%$, and $26 \%$, respectively. Because of the fact that the stationary planetary waves are local time independent, we don't have to consider the damping due to the LT binning. Hence the stationary planetary waves with zonal wavenumber $m=1,2,3,4$ are reduced by $1 \%, 4 \%$, $10 \%, 17 \%$, respectively, considering our analysis approach. Tidal components presented in this paper are corrected for the amplitude alternation.

One goal of this work is to present the annual variation of the observed wave- 4 structure initially detected in the zonal wind during equinoxes by Häusler et al. (2007). For accomplishing this, we made use of 4 years of data taken by the accelerometer onboard the CHAMP satellite. The wave- 4 structure is present throughout the year but varies in strength due to the annual variation of DE3. On the basis of the huge data set available for this study we were able to calculate the individual tidal components embedded in the zonal wind. Thereby, we identified the eastward propagating diurnal tide with zonal wavenumber $s=3$ (DE3) as the primary cause for the observed wave- 4 structure in the zonal wind at $400 \mathrm{~km}$ altitudes. Thus the annual variation of the observed wave- 4 structure and DE3 are quite similar. 
Another prominent nonmigrating tide revealed in the CHAMP zonal wind is the eastward propagating diurnal tide with zonal wavenumber $s=2$ (DE2). Comparing the annual variation of DE2 and DE3 at $400 \mathrm{~km}$ altitude with those for the zonal wind at $100 \mathrm{~km}$ altitude derived from SABER and TIDI measurements onboard the TIMED satellite (Pedatella et al., 2008), we find a big resemblance of the annual variation between the two altitude levels. The tidal components of Pedatella et al. (2008) (their Fig. 1) represent a five-year mean from 2002-2006. Besides the good agreement between the zonal wind at $100 \mathrm{~km}$ and $400 \mathrm{~km}$ altitude, there is also good agreement with the findings of Forbes et al. (2008) who analyzed SABER temperatures for tidal signatures. The authors could confirm a strong DE3 tidal signal for the months of June through October. Also their DE2 tidal temperatures show a similar annual variation as presented here for the DE2 tidal winds of CHAMP.

Both DE3 and DE2 are excited in the tropical troposphere due to latent heat release in deep convective clouds (Hagan and Forbes, 2002). The good qualitative agreements between the MLT and upper thermospheric tidal signals raise the question of how the tidal signatures from the MLT region are coupled to the upper thermosphere. Modeling studies performed by Hagan et al. (2007) with the thermosphereionosphere-mesosphere-electrodynamics general circulation model (TIME-GCM) suggest that at least DE3 is capable of propagating well into the upper thermosphere. Using a physics-based empirical fit model based on Hough Mode Extensions (HMEs), Oberheide and Forbes (2008) are able to extend TIMED tidal observations into the upper thermosphere thus closing the gab between the MLT and measurements taken at $\sim 400 \mathrm{~km}$ altitude. In their study, Oberheide and Forbes (2008) report that at least 50\% of the CHAMP DE3 tidal signal in the zonal wind can be ascribed to direct tidal upward propagation from the troposphere. Revisiting the subject in a more recent study, Oberheide et al. (2009) report that within the limits of uncertainties the DE3 tidal winds at CHAMP altitude are mainly attributable to troposphere forcing. However, this leads to the question why stronger DE3 tidal winds are observed along the geomagnetic equator compared to the geographic equator. Naturally, if DE3 is merely propagating upward from the lower atmosphere it should be strongest along the geographic equator because deep convective clouds are not controlled by the geomagnetic field. Therefore, there has to be an additional connection between the DE3 tidal winds with F-region electrodynamics also displaying wave-4 structures. This link cannot be explained right now. Artificially enhanced DE3 amplitudes along the geomagnetic equator due to the applied data processing have been ruled out by test data sets.

In general, global-scale secondary waves are excited, e.g. through nonlinear interaction between tides and planetary waves. Thereby, the primary waves with frequencies, $f_{P 1}$ and $f_{P 2}$, and zonal wavenumbers, $s_{P 1}$ and $s_{P 2}$, generate secondary waves with frequencies, $f_{S}$, and zonal wavenum- bers, $s_{S}$, so that we get $f_{S}=f_{P 1} \pm f_{P 2}$ and $s_{S}=s_{P 1} \pm s_{P 2}$ (Hagan and Roble, 2001). Numerical experiments with the TIME-GCM model developed at the National Center for Atmospheric Research (NCAR) revealed that the nonlinear interaction between the migrating diurnal tide (DW1) and the DE3 excites a stationary planetary wave-4 oscillation (sPW4) (Hagan et al., 2009). A similar interaction between DW1 and DE2 would yield from theory a stationary planetary wave3 oscillation (sPW3) and an eastward propagating semidiurnal tide with zonal wavenumber $s=1$ (SE1). In Fig. 4 we show, both SE1 and sPW3 display the same annual variation as DE2, suggesting that the aforementioned components could be caused by the nonlinear interaction between DW1 and DE2. However, a comparison of the CHAMP tidal wind signals at $400 \mathrm{~km}$ altitude with TIME-GCM shows that so far the model does not predict the observed DE2 properly (Häusler et al., 2009). Hence, a possible connection between DE2 and SE1 and sPW3 cannot be confirmed yet.

Discernable in Fig. 4 is a strong stationary planetary wave with zonal wavenumber $m=1$ (sPW1). The interaction of sPW1 with the diurnal migrating tide (DW1) generates DW2 and D0 according to the aforementioned relation. Looking at the upper right panel of Fig. 4, we see a strong DW2 and to some extent D0 when SPW1 is high. The same is true for SW1 and SW3 (middle right panel of Fig. 4) which are generated when SPW1 is interacting with SW2. However, the interaction of DW2 and D0 with DW1 yields SW3 and SW1 as well. Therefore, our results alone cannot explain beyond doubt how the individual tidal components are generated and whether they propagate upward from lower altitudes or are excited in-situ. We can only quantify the presence of these nonmigrating tides. Numerical experiments, performed e.g. with TIME-GCM, are needed to further investigate the tidal dynamics in the upper thermosphere.

\section{Summary and conclusions}

In this study we present the annual variation of the prominent wave- 4 structure in the zonal wind at $400 \mathrm{~km}$ altitudes. Analyzing 4 years of CHAMP accelerometer measurements taken between 2002-2005 makes this possible.

The wave-4 is strongest from July through October with a smaller maximum in March and April. During these months, the wave- 4 is propagating $90^{\circ}$ to the east within $24 \mathrm{~h} \mathrm{LT}$.

We are furthermore able to calculate the individual tidal components and show that the observed wave- 4 structure in the upper thermospheric zonal wind can be attributed to the presence of the eastward propagating diurnal tide with zonal wavenumber $s=3$ (DE3). All the other possible contributions to the wave- 4 are rather weak. The identified annual variation (strong maximum in July-October and a smaller one in March/April) corresponds well with tidal properties in the MLT region. However, the observed larger amplitudes along the geomagnetic equator still need to be explored. We 
suppose that there is an additional interaction between the DE3 tidal winds and F-region electrodynamics not explained at the moment.

The eastward propagating diurnal tide with zonal wavenumber $s=2$ (DE2) is comparable in strength with DE3 examined in geographic coordinates but the annual variation is different showing maxima in June and November. However, this annual variation is also consistent with DE2 tidal variations in the MLT. A possible connection between DE2 and SE1 and SPW3, displaying the same annual variation as DE2, cannot be confirmed yet.

Furthermore, we observe strong DW2, D0, SW3, SW1, and SPW1, all contributing to wave-1 in the satellite frame, mostly along the geomagnetic equator.

The presence of these waves in the upper thermosphere, however, cannot be explained without any doubt, calling for model simulations to support the interpretation of the signals. A consequent next step is thus to perform simulations to delineate the coupling mechanisms and to resolve the open questions of tidal waves in the upper atmosphere. The initiated comparison between CHAMP observations and TIMEGCM tidal signals by Häusler et al. (2009) can be regarded as a first step.

Notably, we find that the applied data processing induces a damping of the tidal amplitudes which can be as high as $20 \%$ and $26 \%$ for the diurnal and semidiurnal tides observed as wave- 4 in the satellite frame.

Here we limited our analysis to latitude bands of $\pm 10^{\circ}$ along the geomagnetic and geographic equator. Extending the study to mid latitudes will be the topic of a follow-on study.

Acknowledgements. The operational support of the CHAMP mission by the German Aerospace Centre (DLR) and the financial support for the data processing by the Federal Ministry of Education and Research (BMBF), as part of the Geotechnology Programme, are gratefully acknowledged. One of the authors $(\mathrm{KH})$ is supported by the Deutsche Forschungsgemeinschaft (DFG) Priority Program "CAWSES", SPP 1176.

Topical Editor K. Kauristie thanks K. Cahoy and another anonymous referee for their help in evaluating this paper.

\section{References}

Chapman, S. and Lindzen, R. S.: Atmospheric Tides: Thermal and Gravitational. D. Reidel Publishing Company, Dordrecht, Holland, 1970.

England, S. L., Maus. S., Immel, T. L., and Mende, S. B.: Longitude variation of the E-region electric fields caused by atmospheric tides, Geophys. Res. Lett., 33, L21105, doi:10.1029/2006GL027465, 2006.

Forbes, J. M., Zhang, X., Palo, S., Russell, J., Mertens, C. J., and Mlynczak, M.: Tidal variability in the ionospheric dynamo region, J. Geophys. Res., 113, A02310, doi:10.1029/2007JA012737, 2008.
Forbes, J. M., Russell, J., Miyahara, S., Zhang, X., Palo, S., Mlynczak, M., Mertens, C. J., and Hagan, M. E.: Tropospherethermosphere tidal coupling as measured by the SABER instrument on TIMED during July-September 2002, J. Geophys. Res., 111, A10S06, doi:10.1029/2005JA011492, 2006.

Forbes, J. M., Hagan, M. E., Miyahara, S., Miyoshi, Y., and Zhang, $\mathrm{X}$.: Diurnal nonmigrating tides in the tropical lower thermosphere. Earth Planets Space, 55, 419-426, 2003.

Forbes, J. M.: Tidal and Planetary Waves, in: The upper Mesosphere and Lower Thermosphere: A Review of Experiment and Theory, Geophysical Monograph 87, edited by: Johnson, R. M. and Killeen, T. L., AGU, 1995.

Hagan, M. E., Maute, A., and Roble, R. G.: Tropospheric tidal effects on the middle and upper atmosphere, J. Geophys. Res., 114, A01302, doi:10.1029/2008JA013637, 2009.

Hagan, M. E., Maute, A., Roble, R. G., Richmond, A. D., Immel, T. J., and England, S. L.: Connections between deep tropical clouds and the Earth's ionosphere, Geophys. Res. Lett., 34, L20109, doi:10.1029/2007GL030142, 2007.

Hagan, M. E. and Forbes, J. M., Migrating and nonmigrating diurnal tides in the middle and upper atmosphere excited by tropospheric latent heat release, J. Geophys. Res., 107(D24), 4754, doi:10.1029/2001JD001236, 2002.

Hagan, M. E. and Roble, R. G.: Modeling the diurnal tidal variability with the National Center for Atmospheric Research thermosphere-ionosphere-mesosphere-electrodynamics general circulation model. J. Geophys. Res., 106, 24869-24882, 2001.

Hartman, W. A. and Heelis, R. A.: Longitudinal variations in the equatorial vertical drift in the topside ionosphere, J. Geophys. Res., 112, A03305, doi:10.1029/2006JA011773, 2007.

Häusler, K., Lühr, H., Hagan, M. E., Maute, A., and Roble, R. G.: Comparison of CHAMP and TIME-GCM nonmigrating tidal signals in the thermospheric zonal wind, J. Geophys. Res., in review, 2009.

Häusler, K., Lühr, H., Rentz, S., and Köhler, W.: A statistical analysis of longitudinal dependences of upper thermospheric zonal winds at dip equator latitudes derived from CHAMP, J. Atmos. Solar-Terr. Phys., 69, 1419-1430, doi:10.1016/j.jastp.2007.04.004, 2007.

Immel, T. J., Sagawa, E., England, S. L., Henderson, S. B., Hagan, M. E., Mende, S. B., Frey, H. U., Swenson, C. M., and Paxton, L. J.: Control of equatorial ionospheric morphology by atmospheric tides, Geophys. Res. Lett., 33, L15108, doi:10.1029/2006GL026161, 2006.

Kil, H., Oh, S.-J., Kelley, M. C., Paxton, L. J., England, S. L., Talaat, E., Min, K.-W., and Su, S.-Y.: Longitudinal structure of the vertical ExB drift and ion density seen from ROCSAT-1, Geophys. Res. Lett., 34, L14110, doi:10.1029/2007GL030018, 2007.

Lin, C. H., Wang, W., Hagan, M. E., Hsiao, C. C., Immel, T. J., Hsu, M. L., Liu, J. Y., Paxton, L. J., Fang, T. W., and Liu, C. H.: Plausible effect of atmospheric tides on the equatorial ionosphere observed by the FORMOSAT-3/COSMIC: Three-dimensional electron density structures, Geophys. Res. Lett., 34, L11112, doi:10.1029/2007GL029265, 2007.

Liu, H., Lühr, H., Watanabe, S., Köhler, W., Henize, V., and Visser, P.: Zonal winds in the equatorial upper thermosphere: decomposing the solar flux, geomagnetic activity, and seasonal dependencies, J. Geophys. Res., 111, A07307, doi:10.1029/2005JA011415, 2006. 
Lowes, F. J. and Olsen, N.: A more realistic estimate of the variances and systematic errors in spherical harmonic geomagnetic field models, Geophys. J. Int., 157, 1027-1044, doi: 10.1111/j.1365-246X.2004.02256.x, 2004.

Lühr, H., Rother, M., Häusler, K., Alken, P., and Maus, S.: The influence of nonmigrating tides on the longitudinal variation of the equatorial electrojet, J. Geophys. Res., 113, A08313, doi:10.1029/2008JA013064, 2008.

Lühr, H., Häusler, K., and Stolle, C.: Longitudinal variation of $\mathrm{F}$ region electron density and thermospheric zonal wind caused by atmospheric tides, Geophys. Res. Lett., 34, L16102, doi:10.1029/2007GL030639, 2007.

McLandress, C. and Ward, W. E.: Tidal/gravity wave interactions and their influence on the large-scale dynamics of the middle atmosphere: Model results, J. Geophys. Res., 99, 8139-8155, 1994.

Müller, S., Lühr, H., and Rentz, S.: Solar and magnetospheric forcing of the low latitude thermospheric mass density as observed by CHAMP, Ann. Geophys., 27, 2087-2099, 2009,

http://www.ann-geophys.net/27/2087/2009/.
Oberheide, J., Forbes, J. M., Häusler, K., Wu, Q., and Bruinsma, S. L.: Tropospheric tides from $80-400 \mathrm{~km}$ : propagation, interannual variability and solar cycle effects, J. Geophys. Res., in review, 2009.

Oberheide, J. and Forbes, J. M.: Tidal propagation of deep tropical cloud signatures into the thermosphere from TIMED observations, Geophys. Res. Lett., 35, L04816, doi:10.1029/2007GL032397, 2008.

Oberheide, J., Hagan, M. E., Roble, R. G., and Offermann, D.: Sources of nonmigrating tides in the tropical middle atmosphere. J. Geophys. Res., 107(D21), 4567, doi:10.1029/2002JD002220, 2002.

Pedatella, N. M., Forbes, J. M., and Oberheide, J.: Intra-annual variability of the low-latitude ionosphere due to nonmigrating tides, Geophys. Res. Lett., 35, L18104, doi:10.1029/2008GL035332, 2008.

Sagawa, E., Immel, T. J., Frey, H. U., and Mende S. B.: Longitudinal structure of the equatorial anomaly in the nighttime ionosphere observed by IMAGE/FUV, J. Geophys. Res., 110, A11302, doi:10.1029/2004JA010848, 2005. 